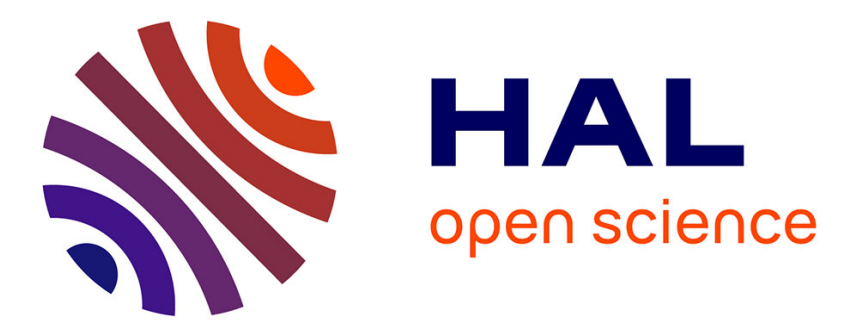

\title{
Influence of Dissipated Energy on Shear Band Spacing in HY100 Steel
}

Omar Oussouaddi, Loïc Daridon, Saïd Ahzi, André Chrysochoos

\section{To cite this version:}

Omar Oussouaddi, Loïc Daridon, Saïd Ahzi, André Chrysochoos. Influence of Dissipated Energy on Shear Band Spacing in HY100 Steel. Journal of Engineering Materials and Technology, 2011, 133 (2), pp.021002. 10.1115/1.4001592 . hal-00832743

\section{HAL Id: hal-00832743 \\ https://hal.science/hal-00832743}

Submitted on 22 Sep 2016

HAL is a multi-disciplinary open access archive for the deposit and dissemination of scientific research documents, whether they are published or not. The documents may come from teaching and research institutions in France or abroad, or from public or private research centers.
L'archive ouverte pluridisciplinaire HAL, est destinée au dépôt et à la diffusion de documents scientifiques de niveau recherche, publiés ou non, émanant des établissements d'enseignement et de recherche français ou étrangers, des laboratoires publics ou privés. 


\section{Oussouaddi}

EMMS,

Faculté des Sciences et Techniques,

Université My Ismaïl,

Errachidia 52000, Morocco

\section{Daridon}

LMGC-UMR CNRS 5508,

Université Montpellier II,

CC 048,

Montpellier 34095, France

\section{S. Ahzi ${ }^{1}$}

IMFS-UMR CNRS n 7507 ,

Université Louis Pasteur,

2 Rue Boussingault,

Strasbourg 67000, France

e-mail: ahzi@unistra.fr

\section{A. Chrysochoos \\ LMGC-UMR CNRS 5508 , \\ Université Montpellier II, \\ CC 048, \\ Montpellier 34095, France}

${ }^{1}$ Corresponding author.

\section{Influence of Dissipated Energy on Shear Band Spacing in HY-100 Steel}

To analyze the formation of multiple shear bands in HY-100 steel, we consider an infinitely extended layer of finite thickness subjected to shear loading. The perturbation approach, associated with numerical methods, is used to determine the instability modes. The criteria of Wright-Ockendon and Molinari are used to determine the shear band spacing. The hypothesis consisting in considering the proportion of plastic work dissipated as heat (quantified by the Taylor-Quinney coefficient $\beta$ ) as independent of the loading path is now recognized as highly simplistic. The present study attempts to provide a systematic approach to the inelastic heat fraction evolution for a general loading within the framework of thermoviscoplastic standard modeling, including a number of material parameters as strain hardening, strain rate sensitivity, and thermal softening. The effect of each material parameter on the shear band spacing is determined by using a power law constitutive relation. The Johnson-Cook and power law models are used to illustrate the influence of the constitutive relation on the results for the adiabatic shear band spacing by studying the response of HY-100 steel. We have compared our results with available experimental results in the literature over a very wide range of strain rates $\left(10^{3}-10^{5} s^{-1}\right)$. In this study, we show that the variation in the Taylor-Quinney parameter $\beta(\gamma)$ as a function of shear strain is an important parameter that plays a significant role in the calculation of the shear band spacing.

Keywords: adiabatic shear bands, dissipated energy, perturbation method, shear band spacing, HY-100 steel

\section{Introduction}

Adiabatic shear bands (ASBs) refer to a very narrow area where an intense shear deformation accompanied by a rise in temperature is formed. Tresca [1] was the first who observed hot lines, now called ASBs and concluded that at least $70 \%$ of the plastic working was converted into heating. Taylor and Quinney [2] found that the fraction of the plastic work converted into heat depends upon the material; this fraction lies between $85 \%$ and 95\%. Chrysochoos and Belmahjoub [3] and Mason et al. [4] asserted that the fraction of the plastic working converted into heating depends upon the plastic strain and the plastic strain rate. Zener and Hollomon [5] proposed that the basic mechanism for the formation of ASBs is a dynamic instability caused by adiabatic heating. During the past 20 years, there has been a growing interest in the ASB phenomenon because it often precedes dynamic shear fracture and is the primary mode of failure in ductile materials under sufficiently high-rate dynamic loading. For a detailed review of this topic, see the work of Armstrong et al. [6], Batra and Zbib [7], Bai and Dodd [8], and Wright [9].

In some circumstances, many small bands may form throughout a volume of the material, in which case a spread weakening occurs with the possibility of multiple failures and a general fragmentation. In other circumstances, one band may dominate, and therefore, the material failure is restricted to just that one location.

Grady and co-worker $[10,11]$ obtained the shear band spacing by accounting for momentum diffusion due to unloading within bands. Wright and Ockendon [12] used a perturbation analysis to characterize a dominant mode corresponding to the most probable minimum spacing of shear bands. Thus, the wavelength of the dominant instability mode with the maximum initial growth rate will determine the shear band spacing. Molinari [13] extended the work of Wright and Ockendon [12] to strain hardening materials and has estimated the error in the shear band spacing due to the finite thickness of a block deformed in simple shear. He showed that the shear band spacing increases with an increase in the strain hardening exponent. Batra and Chen [14-16] studied the effect of thermal conductivity on shear band spacing and also showed that it depends on the stress-strain relation used to describe the material behavior. Daridon et al. [17] showed that the mechanical threshold stress model predicts well the value of the shear band spacing in the case of HY-100 steel and Ti-6Al-4V alloy. Recently, Batra and Wei [18] obtained a closed-form expression for the shear band spacing for a strain hardening, strain rate hardening, and thermally softening thermoviscoplastic material deformed under simple shear. More recently, Lapovok et al. [19] used a constitutive description that includes second-order gradient terms in conjunction with the perturbation approach to account for the shear bands formed in the equal-channel angular pressing of magnesium alloy AZ31. They demonstrated that the shear band spacing predicted by linear perturbation analysis is in good agreement with the experimental value. The main objective of this study is to study the influence of the Taylor-Quiney parameter on shear band spacing. We propose a way to take into account the influence of the shear strain on the Taylor-Quinney coefficient.

This study is organized as follows. In Sec. 2, the system of governing equations for one-dimensional simple shearing deformation is formulated. In Sec. 3, we introduce the thermoviscoplastic constitutive laws used in this study. The perturbation method to find the instability strain and the shear band spacing is presented in Sec. 4. If we take into account the fact that the fraction of the plastic work converted into heat depends on the shear strain, we show that this has a considerable influence on spacing between bands. We use a power law constitutive relation to analyze the 


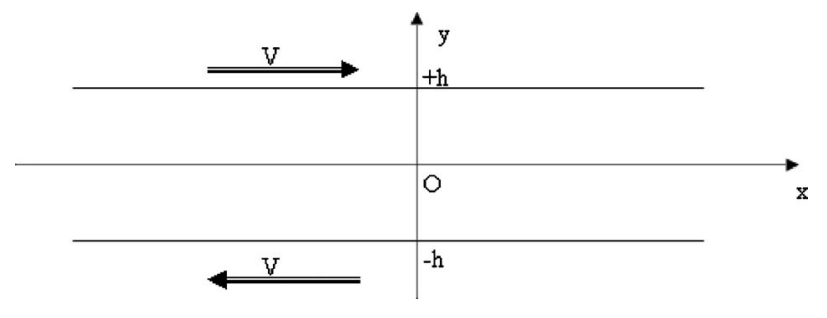

Fig. 1 Geometry used for shear analysis

effect of each material parameter on the shear band spacing. To study the influence of the constitutive model on the results of the adiabatic shear band spacing, we compare the results from the power law and the Johnson-Cook model [20]. At low strain rates, the assumption of a constant Taylor-Quinney parameter leads to a significant underestimation of the shear band spacing.

\section{Formulation of the Problem}

In this study, we consider a layer with finite thickness $2 h$ in the $y$ direction, whereas it is infinite in the shear $x$ direction and in the out-of-plane $z$ direction (Fig. 1). At the upper and lower surfaces, constant velocities $\pm V$ are applied, parallel to the $x$ direction. We assume that all physical quantities are uniform along the $x$ and $z$ directions so that the deformation depends only on the space coordinate $y$, which leads to the vanishing convection term in the heat equation. We neglect the elastic effects, we assume incompressibility, and we consider adiabatic conditions at the boundaries. Therefore, the governing equations are given by

$$
\begin{gathered}
\rho \frac{\partial v}{\partial t}=\frac{\partial \tau}{\partial y} \\
\rho c \frac{\partial T}{\partial t}-k \frac{\partial^{2} T}{\partial y^{2}}=\beta(\gamma) \tau \dot{\gamma} \\
\dot{\gamma}=\frac{\partial v}{\partial y}
\end{gathered}
$$

Equation (1) is the momentum balance, relation (2) is the energy equation, and relation (3) is the compatibility equation. Here, $v, \dot{\gamma}$, $\tau$, and $T$ are, respectively, the particle velocity, the plastic shear strain rate, the shear stress, and the absolute temperature. The parameters $\rho, c, k$, and $\beta(\gamma)$ are, respectively, the mass density, the specific heat, the thermal conductivity, and the TaylorQuinney coefficient, which represents the fraction of the plastic work converted into heat. To model the evolution of the fraction of the plastic work converted into heat, according to the shear deformation, we propose the following empirical relation:

$$
\beta(\gamma)=1-\beta_{0} e^{-\beta_{1} \gamma}
$$

Using the experimental results (see Fig. 2) of Chrysochoos and Belmahjoub [3] obtained for quasi-static loading, the constants $\beta_{0}$ and $\beta_{1}$ are calculated by simple fitting. As $\beta_{0}$ is imposed by the initial condition at zero shear strain, $\beta_{1}$ can be easily found by fitting the rest of the curve. The values of these parameters are respectively equal to 0.45 and 10 .

In order to complete the problem description, we need to introduce a material constitutive relation expressed in the following general form:

$$
\tau=\tau(\gamma, \dot{\gamma}, T)
$$

The initial conditions are given by

$$
v(y, 0)=0, \quad T(y, 0)=T_{i}, \quad \tau(y, 0)=0
$$

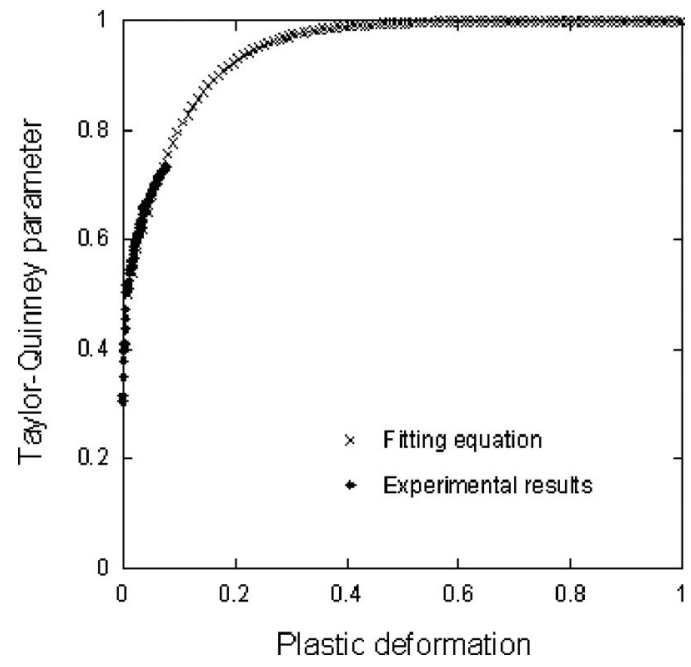

Fig. 2 Experimental and theoretical Taylor-Quinney parameter as function the plastic strain

- A constant velocity $V$ parallel to the shear direction is applied at the boundaries

$$
v(-h, t)=-V, \quad v(+h, t)=+V \quad \text { for } t>0
$$

- At high strain rates, which are considered in this study, adiabatic conditions are assumed at the boundaries

$$
\frac{\partial T}{\partial y}(-h, t)=0 \quad \text { and } \quad \frac{\partial T}{\partial y}(+h, t)=0 \text { for } t>0
$$

In the above equations, $t$ represents time.

\section{Viscoplastic Constitutive Relations}

It is well documented that the existence of a maximum on the stress-strain curve is a necessary condition for the occurrence of adiabatic shear bands. This maximum is due to the competition between the stabilizing effect of the hardening and the strain rate and the destabilizing effect of the thermal softening. Therefore, we must use a thermoviscoplastic relation, which takes these three effects into account. In this study, we will use the power law and the Johnson-Cook models and compare results from both models to the available experimental results in the literature.

3.1 Power Law. In order to analyze the influence of each material parameter on the shear band spacing, it is useful to use a constitutive relation with a simple form and decoupled terms for defining the strain hardening, strain rate hardening, and thermal softening behaviors of the material. Therefore, we used in this study the power law as reference behavior. Different authors, Molinari and Clifton [21], Molinari [22], and Batra and Chen [15], described the stress-strain curves for dynamic loading by

$$
\tau(\gamma, \dot{\gamma}, T)=\tau_{0}\left(\frac{\gamma}{\gamma_{0}}\right)^{n}\left(\frac{\dot{\gamma}}{\dot{\gamma}_{0}}\right)^{m}\left(\frac{T}{T_{0}}\right)^{\nu}
$$

where $\tau_{0}$ is the yield stress of the material in a quasi-static simple shear test, $n$ and $m$ characterize the strain and strain rate hardening of the material and $\nu<0$ is its thermal softening. $\dot{\gamma}_{0}$ is a reference shear rate. $T_{0}$ is a reference temperature and $T$ is the current temperature. $\gamma_{0}$ is the strain at which the stress level $\tau=\tau_{0}$, provided that $\dot{\gamma}=\dot{\gamma}_{0}$ and $T=T_{0}$. For the HY-100 steel, the power law model parameters are given in Table 1 [15].

3.2 Johnson-Cook model. Johnson and Cook [20] proposed a phenomenological model for metals subjected to large strains, high strain rates, and high temperatures. The Johnson-Cook 
Table 1 Power law parameters for HY-100 steel [15]

\begin{tabular}{lccccccr}
\hline \hline Parameter & $\begin{array}{c}\tau_{0} \\
(\mathrm{MPa})\end{array}$ & $\gamma_{0}$ & $\begin{array}{c}\dot{\gamma}_{0} \\
\left(\mathrm{~s}^{-1}\right)\end{array}$ & $\begin{array}{c}T_{0} \\
(\mathrm{~K})\end{array}$ & $n$ & $m$ & $\nu$ \\
\hline HY-100 & 405 & 0.012 & 3300 & 300 & 0.107 & 0.0117 & 0.75 \\
\hline \hline
\end{tabular}

model has enjoyed much success because of its simplicity and the availability of parameters of various materials of interest. This model expresses the flow shear stress by

$$
\tau(\gamma, \dot{\gamma}, T)=\left(A+B \gamma^{N}\right)\left(1+C \ln \left(\frac{\dot{\gamma}}{\dot{\gamma}_{0}}\right)\right)\left(1-\left(\frac{T-T_{0}}{T_{m}-T_{0}}\right)^{M}\right)
$$

where $\gamma$ is the plastic shear strain and $\dot{\gamma}_{0}$ is a reference shear strain rate. $T_{0}$ and $T_{m}$ are, respectively, the initial (or the reference) temperature and the melting temperature. The coefficients $A, B, N$, $M$, and $C$ are constitutive parameters. In the right hand side of Eq. (10), the first term gives the stress as a function of the strain hardening coefficient $B$ and the strain hardening exponent $N$; the second term represents instantaneous strain rate sensitivity; and the last term represents the temperature dependence of the flow stress. Here, $C$ is the strain rate parameter and $M$ is the thermal softening parameter. The Johnson-Cook model parameters for the HY-100 steel are given in Table 2 [15].

\section{Perturbation Analysis}

We consider the homogeneous solutions $\tau^{(0)}(t), v^{(0)}(t), \gamma^{(0)}(t)$, and $T^{(0)}(t)$ for shear stress, velocity, shear strain, and temperature, respectively. The stability of the homogeneous solution is analyzed by adding a small perturbation to the basic solution at a time specified by $t_{0}$ (see for instance Refs. $[13,16]$ )

$$
\delta s\left(y, t, t_{0}\right)=\delta s^{(0)} e^{\eta\left(t-t_{0}\right)} e^{i \xi y}, \quad t \geq t_{0}
$$

where $\delta \mathbf{s}^{(0)}=\left(\delta v^{(0)}, \delta \gamma^{(0)}, \delta \tau^{(0)}, \delta T^{(0)}\right)$ and $y$ is the position along the layer thickness. The quantities $\delta v^{(0)}, \delta \gamma^{(0)}, \delta \tau^{(0)}$, and $\delta T^{(0)}$ characterize the amplitude at time $t_{0}$ of the perturbation. The parameter $\xi$ is the wave number of the perturbation and $\eta$ is the inverse of the characteristic time, called growth rate of the perturbation. The fundamental solution is stable when the real part of $\eta$ is negative, $R_{e}(\eta)<0$, and unstable when $R_{e}(\eta)>0$.

The form of the perturbed solution is given by

$$
s\left(y, t, t_{0}\right)=s^{(0)}(y, t)+\delta s\left(y, t, t_{0}\right)
$$

Here, $s=(v, \gamma, \tau, T)$. By substituting the solution (Eq. (11)) into the governing equations (1)-(5) and after a linearization in $\delta \mathbf{s}^{0}$, we get

$$
\mathbf{A}\left(t_{0}, \eta, \xi\right) \cdot \delta \mathbf{s}^{0}=0
$$

This set of linear equations admits a nontrivial solution only if the determinant of the matrix $\mathbf{A}$ is equal to zero. This leads to a cubic equation for the growth rate $\eta$ of the perturbation

$$
\begin{gathered}
\rho^{2} c \eta^{3}+\rho\left(\left.c \xi^{2} \frac{\partial \tau}{\partial \dot{\gamma}}\right|_{s^{0}}+k \xi^{2}-\left.\beta^{0} \dot{\gamma}^{0} \frac{\partial \tau}{\partial T}\right|_{s^{0}}\right) \eta^{2}+\left(\left.k \xi^{2} \frac{\partial \tau}{\partial \dot{\gamma}}\right|_{s^{0}}\right. \\
\left.+\left.\rho c \frac{\partial \tau}{\partial \gamma}\right|_{s^{0}}+\left.\beta^{0} \tau^{0} \frac{\partial \tau}{\partial T}\right|_{s^{0}}\right) \xi^{2} \eta+\left.k \xi^{4} \frac{\partial \tau}{\partial \gamma}\right|_{s^{0}}=0
\end{gathered}
$$

Here, the partial derivatives are evaluated for the fundamental solution at time $t_{0}$ and $\beta\left(\gamma^{0}\right)=\beta^{0}$. For the given values of $\gamma^{0}$ $=\gamma^{0}\left(t_{0}\right)$ and $\xi$, three complex roots are obtained, $\eta_{i}\left(\xi, \gamma^{0}\right)(i$ $=1,2,3)$. The root with the largest positive real part governs the instability of the homogeneous solution, and is hereafter referred to as the dominant instability mode (denoted $\eta_{D}$ ).

We consider that the zone where instability is expected to be sufficiently narrow for deformation therein to be treated as homogeneous. The fundamental solution is such that the strain rate is uniform, $\dot{\gamma}^{0}=V / h$. The temperature can be obtained by the integration of the heat equation (2), where the diffusion term vanishes, and where the constitutive law (Eq. (9) or (10)) is used to express the stress $\tau$.

\section{Results and Discussions}

For the HY-100 steel modeled by the power law equation (9), Fig. 3 shows the dominant growth rate $\eta_{D}$ versus the wave number $\xi$ for various values of the average strain. These curves have been computed for a nominal strain rate $\dot{\gamma}^{0}=10^{4} \mathrm{~s}^{-1}$, and an initial temperature equal to $298 \mathrm{~K}$ for varying the Taylor-Quinney coefficient (Eq. (4)). For each value of the average strain, the dominant growth rate increases until it attains a maximum value (corresponding to $\xi_{c}$ ) and then decreases for large $\xi$. The existence of this maximum is characteristic of the dominant instability mode resulting from the competition of two stabilizing effects: Inertia restrains the growth of long-wavelength modes (small $\xi$ ) while heat conduction restrains the growth of small-wavelength modes (large $\xi$ ). In what follows, the maximum dominant growth rate at time $t_{0}$ for the perturbation is called the critical growth rate $\eta_{c}$, and the corresponding wave number is defined as the critical wave number $\xi_{c}$. Figure 4 shows the dependence of the critical growth rate $\eta_{c}$ and its corresponding wavelength $L_{c}=2 \pi / \xi_{c}$ on the average strain $\gamma^{0}$ for HY-100 steel modeled by the power law. We observe that the curves of the critical growth rate and the critical wavelength versus the average strain have respectively a maximum $\eta_{c m}$ and a minimum $L_{c m}$. These values are obtained for two different values of the average strain $\gamma_{1}^{0}$ and $\gamma_{2}^{0}$. In the example considered here, since the values of $\gamma_{1}^{0}$ and $\gamma_{2}^{0}$ are very close, we assume that $\gamma_{1}^{0} \cong \gamma_{2}^{0}$. This assumption is in good agreement with

\begin{tabular}{|c|c|c|c|c|c|c|c|}
\hline Parameter & $\begin{array}{c}A \\
(\mathrm{MPa})\end{array}$ & $\begin{array}{c}B \\
(\mathrm{MPa})\end{array}$ & $N$ & C & $M$ & $\begin{array}{c}\dot{\gamma}_{0} \\
\left(\mathrm{~s}^{-1}\right)\end{array}$ & $\begin{array}{c}T_{0} \\
(\mathrm{~K})\end{array}$ \\
\hline HY-100 & 182.25 & 580.36 & 0.107 & 0.0227 & 0.7 & 3300 & 300 \\
\hline
\end{tabular}
the results obtained by Molinari [13] in the case of XC18 steel.

Wright and Ockendon [12] postulated that the dominant instability mode with the maximum growth rate at time $t_{0}^{\eta_{c}}$ determines the shear band spacing

Table 2 Johnson-Cook parameters for HY-100 steel [15] 


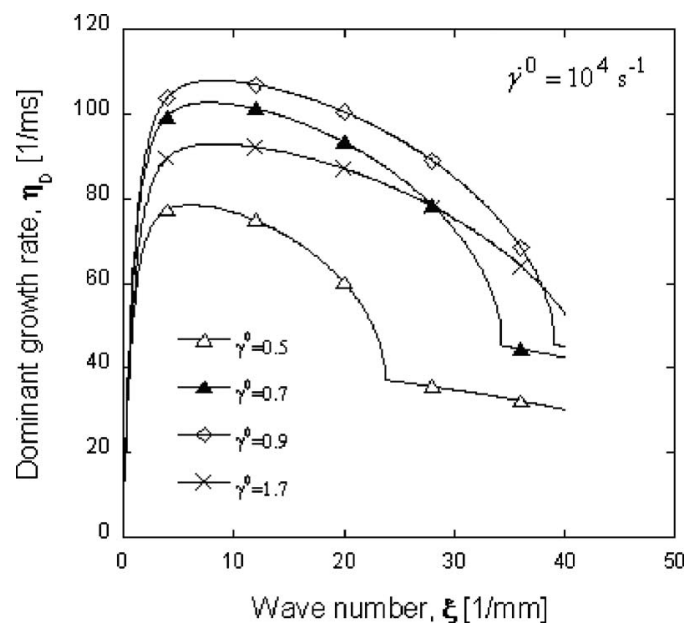

Fig. 3 Dominant growth rate $\eta_{D}$ versus the wave number $\xi$ for HY-100 steel and the power law

$$
L_{s}=\frac{2 \pi}{\xi\left(t_{0}^{\eta_{c}}\right)}
$$

where $t_{0}^{\eta_{c}}$ corresponds to the time when the dominant instability $\eta_{c}\left(t_{0}\right)$ is maximum.

For strain hardening materials, Molinari [13] defined the concept of critical time and corresponding strain, and postulated that the shear banding spacing is given by

$$
L_{s}=\min L_{c}\left(\gamma^{0}\right)=\min \frac{2 \pi}{\xi_{c}\left(\gamma^{0}\right)}
$$

5.1 Effect of Material Parameters. In order to analyze the influence of each material parameter on the shear band spacing, we considered a nominal shear strain rate $\dot{\gamma}^{0}=10^{4} \mathrm{~s}^{-1}$ and an initial temperature $T_{0}=298 \mathrm{~K}$. The results are given in both cases where the Taylor-Quinney coefficient is supposed to be constant $(\beta=0.9)$ and where it evolves with the shear strain (Eq. (4)).

5.1.1 Effect of Strain Hardening. The effect of the strain hardening exponent $n$ on the shear band spacing $L_{S}$ is illustrated in Fig. 5. We used the parameters for HY-100 steel given in Table 1, except for the strain hardening $n$. We note that the shear band spacing increases monotonically with an increasing value of $n$.

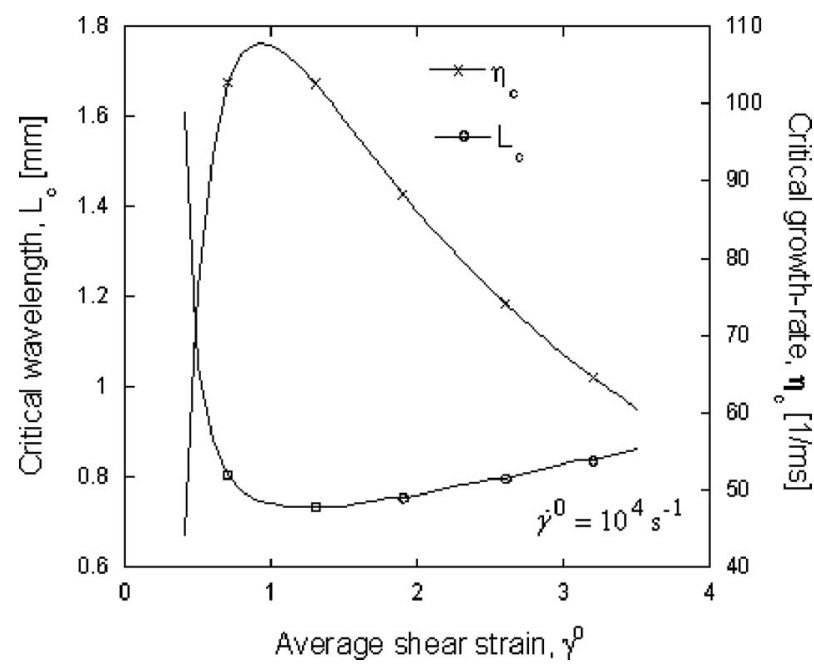

Fig. 4 Influence of $\gamma^{0}$ on $\eta_{c}$ and $L_{c}$ for HY-100 steel and the power law

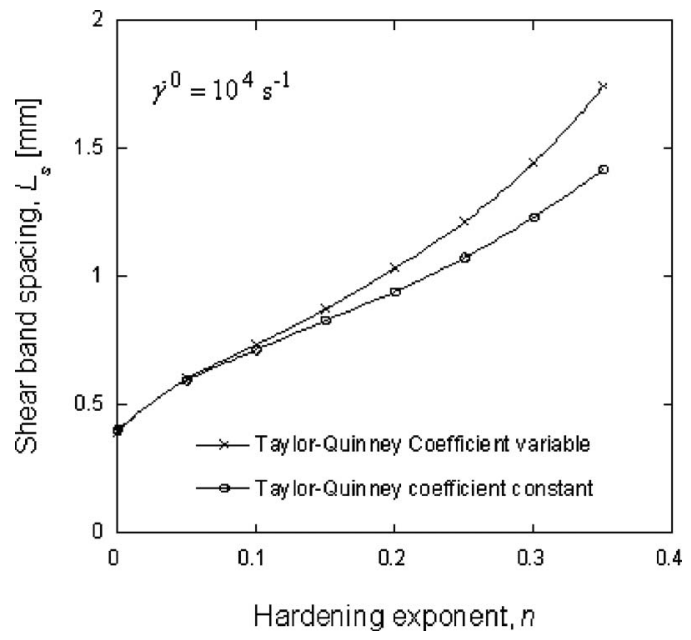

Fig. 5 Influence of the strain hardening exponent $\boldsymbol{n}$ on the shear band spacing $L_{s}$

This is in accord with the known stabilizing work hardening effect. It is noticed that for materials having a high strain hardening exponent, the assumption of a constant coefficient of TaylorQuinney involves an undervaluation of the shear band spacing, particularly for larger values of $n$.

5.1.2 Effect of Strain Rate Sensitivity. It is well known from numerous studies that the rate sensitivity parameter has a stabilizing effect on the flow. Our focus here is the effect on the shear band spacing. To investigate the effects of strain rate sensitivity, we varied the value of the strain rate sensitivity parameter $m$. The strain hardening parameter and the thermal softening parameter were kept constant.

As shown in Fig. 6, an increase in the value of $m$ has a stabilizing effect manifesting itself by a significant increase in the shear band spacing, and hence, a decrease in the number of shear bands. For higher values of $m$, our calculations show a tendency for the shear band spacing to saturate when the Taylor-Quinney coefficient evolves with the shear strain. In this case, the assumption of a constant Taylor-Quinney coefficient involves a noticeable overvaluation of the shear band spacing.

5.1.3 Effect of Thermal Softening. The effects of the thermal softening were investigated by varying the thermal softening parameter $\nu$ between 0.2 and 1.0. The strain hardening exponent and

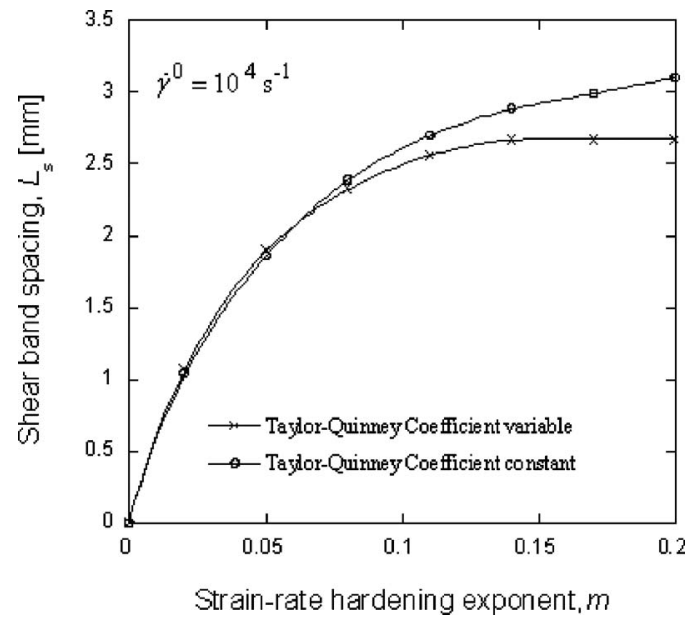

Fig. 6 Influence of the strain rate hardening exponent $m$ on the shear band spacing $L_{s}$ 


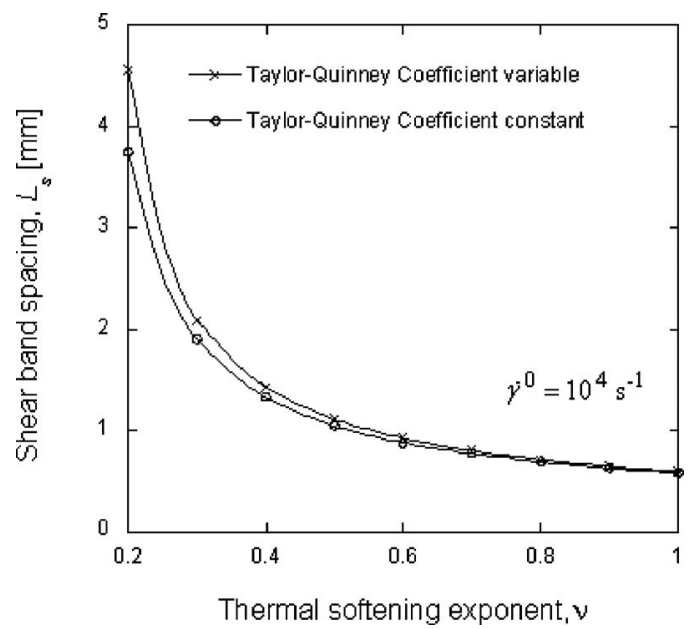

Fig. 7 Influence of the thermal softening exponent $\nu$ on the shear band spacing $L_{s}$

the strain rate sensitivity parameter were kept constant. The results are shown in Fig. 7, where an increase in the softening parameter $\nu$ has a destabilizing effect manifesting itself by a significant decrease in the shear band spacing. For small values of $\nu, L_{s}$ decreases quickly, whereas for the larger values of $\nu, L_{s}$ tends toward a saturation value. Our results in Fig. 7 also show that for materials having low thermal sensitivity, the assumption of the constant Taylor-Quinney coefficient involves a considerable undervaluation of shear band spacing.

5.2 Influence of the Constitutive Relation. Figure 8 shows the dependence of the critical growth rate $\eta_{c}$ and its corresponding wavelength $L_{c}=2 \pi / \xi_{c}$ on the average strain $\gamma^{0}$ for HY-100 steel for the Johnson-Cook model. These curves have been computed for a nominal strain rate $\dot{\gamma}^{0}=10^{4} \mathrm{~s}^{-1}$ and an initial temperature $T_{0}=300 \mathrm{~K}$. The critical growth rate $\eta_{c}$ exhibits a maximum, but the critical wavelength $L_{c}$ has no minimum value. Therefore, the Molinari's postulate does not apply for the Johnson-Cook model. According to Molinari's postulate, the shear band spacing will be essentially zero, whereas the Wright-Ockendon definition gives the value of $L_{s}=1.01 \mathrm{~mm}$ for a constant Taylor-Quinney coefficient, and $L_{s}=0.97 \mathrm{~mm}$ for a varying Taylor-Quinney coefficient.

However, by using the power law and for a varying Taylor-

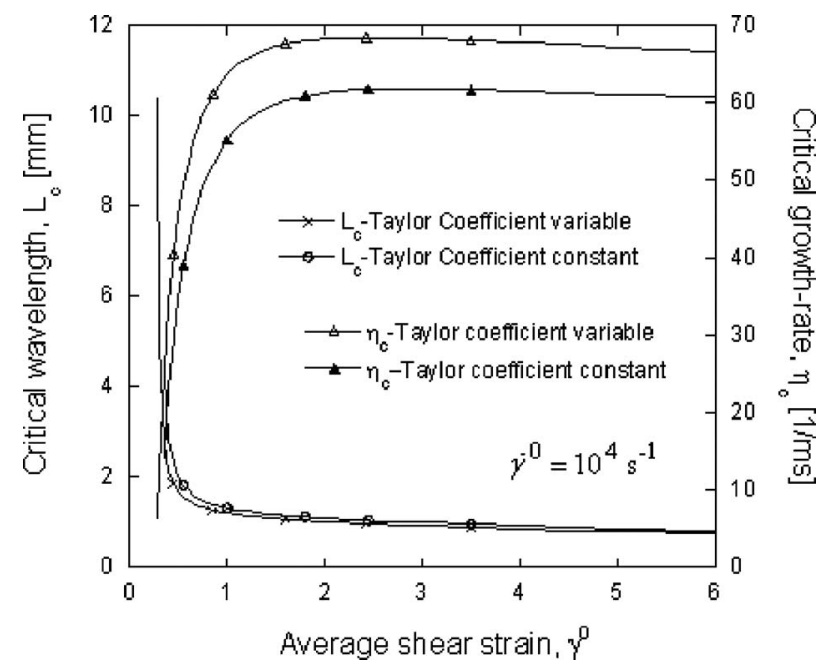

Fig. 8 Influence of $\gamma^{0}$ on $\eta_{c}$ and $L_{c}$, for HY-100 steel and the Johnson-Cook model

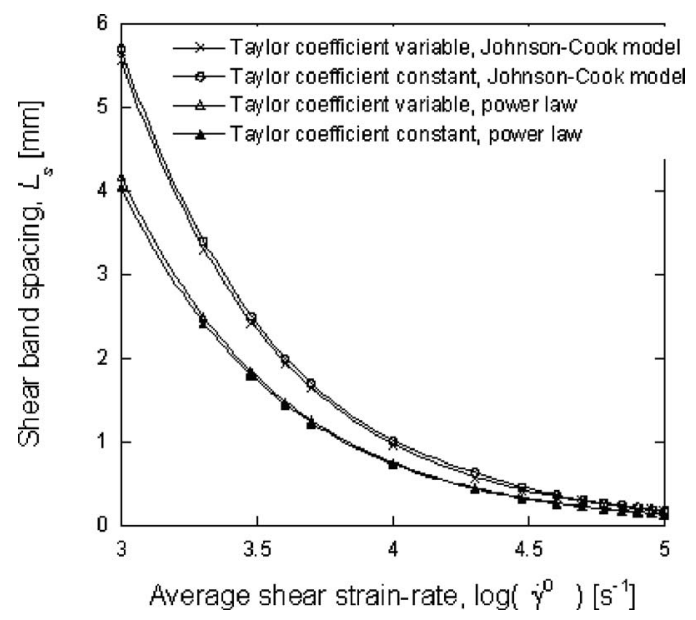

Fig. 9 Influence of the nominal strain rate on the shear band spacing $L_{s}$ for the power law and the Johnson-Cook model

Quinney coefficient, the shear band spacing is equal to $0.73 \mathrm{~mm}$ (see Fig. 4). Consequently, we can conclude that the results obtained with the two models are slightly different and that they are in an acceptable range in comparison with the results presented in the literature, particularly those of Nesterenko et al. [23], where they observed approximately 32 shear bands regularly separated by $1 \mathrm{~mm}$ in the case of an austenitic stainless steel.

We note that there is a limit to the wavelength, which corresponds to the size of the shear zone. The large wavelength perturbation in the current analysis is $12 \mathrm{~mm}^{-1}$ and the corresponding size of the shear band is equal to $0.53 \mathrm{~mm}$. The considered size $(2 h)$ is, in general, equal to $2 \mathrm{~mm}$, and thus, all wavelength perturbations are inside this zone.

5.2.1 Effect of the Shear Strain Rate. For the HY-100 steel modeled by two different constitutive relations (Johnson-Cook and power law), Fig. 9 shows the dependence of the shear band spacing on the average shear strain rate. In both cases where the Taylor-Quinney coefficient is supposed to be constant $(\beta=0.9)$ and where it evolves with the shear strain and for each one of the two models, the shear band spacing decreases rapidly with an increase in the average shear strain rate, and show a tendency to saturate at high strain rates. The difference between the theoretical predictions obtained by the two models is more important for low nominal shear strain. For instance, in the case where the TaylorQuinney coefficient evolves with the shear strain, at $\dot{\gamma}^{0}=10^{3} \mathrm{~s}^{-1}$, the shear band spacing is equal to $4.187 \mathrm{~mm}$ and $5.581 \mathrm{~mm}$, for the power law and the Johnson-Cook models, respectively. On the other hand at $\dot{\gamma}^{0}=10^{5} \mathrm{~s}^{-1}, L_{s}$ is equal to $0.138 \mathrm{~mm}$ and $0.174 \mathrm{~mm}$ for the power law and the Johnson-Cook models, respectively.

We also note that the evolution of the Taylor-Quinney coefficient has an important influence on the shear band spacing $L_{s}$ at relatively low shear strain rate. When it is assumed to be constant, the results lead to significant underestimation of the shear band spacing in the case of the power law, and overestimation in the case of the Johnson-Cook model. Indeed, for the nominal shear strain rate equal to $10^{3} \mathrm{~s}^{-1}$ and the power law, the shear band spacing is equal to $4.051 \mathrm{~mm}$ if we supposed that the coefficient of Taylor is constant, whereas by taking account the experimental results, which show that the Taylor-Quinney coefficient evolves with the shear strain, $L_{s}$ is equal to $4.187 \mathrm{~mm}$.

5.2.2 Effect of the Thermal Conductivity. Several values of thermal conductivity are considered to illustrate its influence on the shear band spacing. The other material parameters remain constant and correspond to those of HY-100 steel. For the power law and the Johnson-Cook model, Fig. 10 shows that the shear band spacing $L_{s}$ increases in a monotonous way with the increase in 


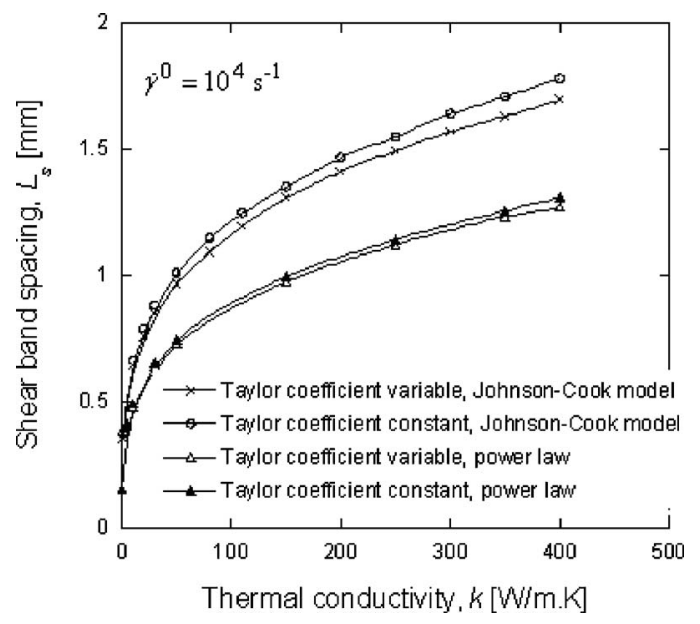

Fig. 10 Influence of the thermal conductivity on the shear band spacing $L_{s}$ for the power law and the Johnson-Cook model

thermal conductivity $k$. This result is in agreement with the stabilizing effect of thermal conductivity. However, the shear band spacing obtained by the Johnson-Cook model is larger than that obtained by the power law, and the gap between the two predictions increases with the thermal conductivity coefficient. Figure 10 shows also an appreciable difference between the results for constant and evolving Taylor-Quinney coefficient, particularly at large values of the thermal conductivity.

\section{Conclusions}

We studied the thermomechanical response of the HY-100 steel block deformed in simple shear. The description of the deformation behavior is modeled using the power law and the JohnsonCook model. The stability of the homogeneous solution is studied by using the perturbation technique.

We have showed that the fraction of plastic work converted into heat may have a significant role in the calculation of the shear band spacing. Indeed, for materials having a high strain hardening exponent, the assumption of a constant coefficient of TaylorQuinney involves an underestimation of the shear band spacing. On the other hand, for a high strain rate sensitivity parameter, we observe a noticeable overestimation of the shear band spacing. It is also noted that for materials having a low thermal sensitivity, the assumption of a constant Taylor-Quinney coefficient involves a considerable underestimation of shear band spacing.

We show that the results obtained with the power law and the Johnson-Cook model are different in the case of low nominal shear strain rate and they are in an acceptable range in comparison with the experimental results. This difference becomes negligible at high nominal shear strain rate.

We also showed that for materials having a high thermal conductivity, it is necessary to correctly account for the evolution of the fraction of plastic work converted into heat.
In any way, the use of a constant value for the inelastic heat fraction constitutes a coarse and conservative simplification, which can sometimes lead to erroneous results. This leads to the necessity for a fine and physically based, thermomechanical reformulation of the constitutive equations used in dynamic loading.

\section{References}

[1] Tresca, H., 1878, "On Further Application of the Flow of Solids," Proc. Inst. Mech. Eng., 29, pp. 301-345.

[2] Taylor, G. I., and Quinney, H., 1934, "The Latent Energy Remaining in a Metal After Cold Working," Proc. R. Soc. London, 143, pp. 307-326.

[3] Chrysochoos, A., and Belmahjoub, F., 1992, "Thermographic Analysis of Thermo-Mechanical Couplings," Arch. Mech., 44, pp. 55-68.

[4] Mason, J. J., Rosakis, A. J., and Ravichandran, G., 1994, "On the Strain and Strain Rate Dependence of the Fraction of Plastic Work Converted to Heat: An Experimental Study Using High Speed Infrared Detectors and Kolsky Bar," Mech. Mater., 17, pp. 135-145.

[5] Zener, C., and Hollomon, J. H., 1944, "Effect of Strain Rate Upon Plastic Flow of Steel," J. Appl. Phys., 15, pp. 22-32.

[6] Armstrong, R., Batra, R. C., Meyers, M., and Wright, T. W., 1994, "Shear Instabilities and Viscoplasticity Theories," Special issue of Mechanics of Materials, 17, pp. 83-328.

[7] Batra, R. C., and Zbib, H. M., 1994, Material Instabilities: Theory and Applications, ASME, New York.

[8] Bai, Y. L., and Dodd, B., 1992, Adiabatic Shear Localization: Occurrence, Theories, and Applications, Pergamon, New York.

[9] Wright, T. W., 2002, The Physics and Mathematics of Adiabatic Shear Bands, Cambridge University Press, Cambridge, England.

[10] Grady, D. E., and Kipp, M. E., 1987, "The Growth of Unstable Thermoplastic Shear With Application to Steady-Wave Shock Compression in Solids," J. Mech. Phys. Solids, 35, pp. 95-119.

[11] Grady, D. E., 1992, "Properties of an Adiabatic Shear-Band Process Zone," J. Mech. Phys. Solids, 40(6), pp. 1197-1215.

[12] Wright, T. W., and Ockendon, H., 1996, "A Scaling Law for the Effect of Inertia on the Formation of Adiabatic Shear Bands," Int. J. Plast., 12, pp. 927-934.

[13] Molinari, A., 1997, "Collective Behavior and Spacing of Adiabatic Shear Bands," J. Mech. Phys. Solids, 45, pp. 1551-1575.

[14] Batra, R. C., and Chen, L., 1999, "Shear Band Spacing in Gradient-Dependent Thermoviscoplastic Materials," Comput. Mech., 23, pp. 8-19.

[15] Batra, R. C., and Chen, L., 2001, "Effect of Viscoplastic Relations on the Instability Strain, Shear Band Initiation Strain, the Strain Corresponding to the Maximum Shear Band Spacing, and the Band Width in a Thermoviscoplastic Material," Int. J. Plast., 17, pp. 1465-1489.

[16] Chen, L., and Batra, R. C., 1999, "Effect of Material Parameters on Shear Band Spacing in Work Hardening Gradient Dependent Thermoviscoplastic Materials," Int. J. Plast., 15, pp. 551-574.

[17] Daridon, L., Oussouaddi, O., and Ahzi, S., 2004, "Influence of the Material Constitutive Models on the Adiabatic Shear Band Spacing: MTS, Power Law and Johnson-Cook Models," Int. J. Solids Struct., 41, pp. 3109-3124.

[18] Batra, R. C., and Wei, Z. G., 2006, "Shear Band Spacing in Thermoviscoplastic Materials," Int. J. Impact Eng., 32, pp. 947-967.

[19] Lapovok, R., Toth, L. S., Molinari, A., and Estrin, Y., 2009, "Strain Localization Patterns Under Equal-Channel Angular Pressing," J. Mech. Phys. Solids, 57, pp. 122-136.

[20] Johnson, G. R., and Cook, W. H., 1983, “A Constitutive Model and Data for Metals Subjected to Large Strains, High Strain-Rates, and High Temperatures," Proceedings of the Seventh International Symposium on Ballistics, pp. 541-547.

[21] Molinari, A., 1985, "Instabilité thermoviscoplastique en cisaillement simple," J. Mec. Theor. Appl., 4, pp. 659-684.

[22] Molinari, A., and Clifton, R., 1983, "Localisation de la déformation viscoplastique en cisaillement simple: résultats exacts en théorie non linéaire," C. R Acad. Sci., Ser. II, 296, pp. 1-4.

[23] Nesterenko, V. F., Meyers, M. A., and Wright, T. W., 1995, "Collective Behavior of Shear Bands," Metallurgical and Materials Application of ShockWave and High-Strain-Rate Phenomena, L. E. Murr, K. P. Staudhammer, and M. A. Meyers, eds., Elsevier, Amsterdam, pp. 397-404. 